\title{
MODELING OF THE HERMES SUBMILLIMETER SOURCE LENSED BY A DARK MATTER DOMINATED FOREGROUND GROUP OF GALAXIES*
}

R. Gavazzi ${ }^{1}$, A. Cooray ${ }^{2,3}$, A. Conley ${ }^{4}$, J. E. Aguirre ${ }^{5}$, A. Amblard ${ }^{6}$, R. Auld ${ }^{7}$, A. Beelen ${ }^{8}$, A. Blain $^{3}$, R. Blundell $^{9}$, J. BocK $^{3,10}$, C. M. Bradford ${ }^{3,10}$, C. Bridge ${ }^{3}$, D. Brisbin ${ }^{11}$, D. Burgarella ${ }^{12}$, P. Chanial ${ }^{13}$, E. Chapin ${ }^{14}$, N. Christopher ${ }^{15}$, D. L. Clements ${ }^{16}$, P. Cox $^{17}$, S. G. Djorgovski ${ }^{3}$, C. D. Dowell ${ }^{3,10}$, S. Eales ${ }^{7}$, L. Earle ${ }^{18}$, T. P. Ellsworth-Bowers ${ }^{4}$, D. Farrah ${ }^{19}$, A. Franceschini ${ }^{20}$, H. Fu ${ }^{3}$, J. Glenn ${ }^{4}$, E. A. GonZÁlez Solares ${ }^{21}$, M. Griffin ${ }^{7}$, M. A. Gurwell ${ }^{9}$,

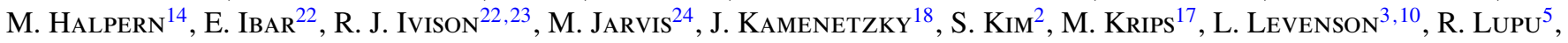
A. Mahabal ${ }^{3}$, P. D. Maloney ${ }^{18}$, C. Maraston ${ }^{25}$, L. Marchetti ${ }^{20}$, G. Marsden ${ }^{14}$, H. Matsuhara ${ }^{26}$, A. M. J. Mortier ${ }^{16}$,

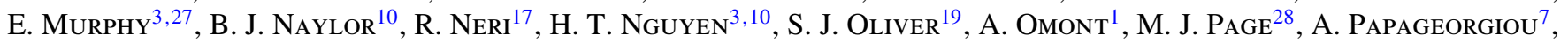
C. P. Pearson ${ }^{29,30}$, I. Pérez-Fournon ${ }^{31,32}$, M. Pohlen $^{7}$, N. Rangwala ${ }^{4}$, J. I. Rawlings ${ }^{28}$, G. Raymond ${ }^{7}$, D. Riechers ${ }^{3,35}$, G. Rodighiero ${ }^{20}$, I. G. Roseboom ${ }^{19}$, M. Rowan-Robinson ${ }^{16}$, B. Schulz ${ }^{3,27}$, Douglas Scott ${ }^{14}$, K. S. Scott ${ }^{5}$, P. Serra ${ }^{2}$, N. Seymour ${ }^{28}$, D. L. Shupe ${ }^{3,27}$, A. J. Smith ${ }^{19}$, M. Symeonidis ${ }^{28}$, K. E. TugWell ${ }^{28}$, M. VACCARi ${ }^{20}$, E. Valiante ${ }^{14}$, I. Valtchanov ${ }^{33}$, A. Verma ${ }^{15}$, J. D. Vieira ${ }^{3}$, L. Vigroux ${ }^{1}$, L. Wang ${ }^{19}$, J. Wardlow ${ }^{2}$, D. Wiebe ${ }^{14}$, G. Wright ${ }^{22}$, C. K. Xu ${ }^{3,27}$,

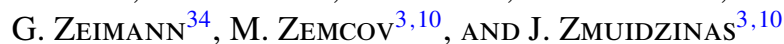

${ }^{1}$ Institut d'Astrophysique de Paris, UMR 7095, CNRS, UPMC Univ. Paris 06, F-75014 Paris, France

${ }^{2}$ Department of Physics \& Astronomy, University of California, Irvine, CA 92697, USA

${ }^{3}$ Astronomy Department, California Institute of Technology, Pasadena, CA 91125, USA

${ }^{4}$ Department of Astrophysical and Planetary Sciences, CASA 389-UCB, University of Colorado, Boulder, CO 80309, USA

${ }^{5}$ Department of Physics and Astronomy, University of Pennsylvania, Philadelphia, PA 19104, USA

${ }^{6}$ NASA Ames Research Center, MS 245-6, Bldg. 245, Moffett Field, CA 94035, USA

${ }^{7}$ Cardiff School of Physics and Astronomy, Cardiff University, Queens Buildings, The Parade, Cardiff CF24 3AA, UK

${ }^{8}$ Institut d'Astrophysique Spatiale (IAS), Université Paris-Sud 11 and CNRS (UMR 8617), 91405 Orsay, France

${ }^{9}$ Harvard-Smithsonian Center for Astrophysics, Cambridge, MA 02138, USA

${ }^{10}$ Jet Propulsion Laboratory, Pasadena, CA 91109, USA

${ }^{11}$ Space Science Building, Cornell University, Ithaca, NY 14853-6801, USA

${ }^{12}$ Laboratoire d'Astrophysique de Marseille, OAMP, Université Aix-Marseille, CNRS, 13388 Marseille cedex 13, France

${ }^{13}$ Laboratoire AIM-Paris-Saclay, CEA/DSM/Irfu-CNRS-Université Paris Diderot, CEA-Saclay, F-91191 Gif-sur-Yvette, France

${ }^{14}$ Department of Physics \& Astronomy, University of British Columbia, Vancouver, BC V6T 1Z1, Canada

${ }^{15}$ Department of Astrophysics, Denys Wilkinson Building, University of Oxford, Oxford OX1 3RH, UK

${ }^{16}$ Astrophysics Group, Imperial College London, Blackett Laboratory, London SW7 2AZ, UK

${ }^{17}$ Institut de RadioAstronomie Millimétrique, Domaine Universitaire, 38406 Saint Martin d'Hères, France

${ }^{18}$ Center for Astrophysics and Space Astronomy, University of Colorado, Boulder, CO 80309, USA

${ }^{19}$ Astronomy Centre, Department of Physics \& Astronomy, University of Sussex, Brighton BN1 9QH, UK

${ }^{20}$ Dipartimento di Astronomia, Università di Padova, 35122 Padova, Italy

${ }^{21}$ Institute of Astronomy, University of Cambridge, Cambridge CB3 OHA, UK

${ }^{22}$ UK Astronomy Technology Centre, Royal Observatory, Blackford Hill, Edinburgh EH9 3HJ, UK

${ }^{23}$ Institute for Astronomy, University of Edinburgh, Royal Observatory, Blackford Hill, Edinburgh EH9 3HJ, UK

${ }^{24}$ Centre for Astrophysics Research, University of Hertfordshire, Hatfield, Hertfordshire AL10 9AB, UK

${ }^{25}$ Institute of Cosmology and Gravitation, University of Portsmouth, Dennis Sciama Building, Portsmouth PO1 3FX, UK

${ }^{26}$ Institute for Space and Astronautical Science, Japan Aerospace and Exploration Agency, Sagamihara, Kanagawa 229-8510, Japan

${ }^{27}$ Infrared Processing and Analysis Center, MS 100-22, California Institute of Technology, JPL, Pasadena, CA 91125, USA

${ }^{28}$ Mullard Space Science Laboratory, University College London, Holmbury St. Mary, Dorking, Surrey RH5 6NT, UK

${ }^{29}$ Space Science \& Technology Department, Rutherford Appleton Laboratory, Chilton, Didcot, Oxfordshire OX11 0QX, UK

${ }^{30}$ Institute for Space Imaging Science, University of Lethbridge, Lethbridge, Alberta T1K 3M4, Canada

${ }^{31}$ Instituto de Astrofísica de Canarias (IAC), E-38200 La Laguna, Tenerife, Spain

${ }^{32}$ Departamento de Astrofísica, Universidad de La Laguna (ULL), E-38205 La Laguna, Tenerife, Spain

${ }^{33}$ Herschel Science Centre, European Space Astronomy Centre, Villanueva de la Cañada, 28691 Madrid, Spain

${ }^{34}$ Department of Physics, University of California, Davis, CA 95616, USA

Received 2011 February 9; accepted 2011 June 7; published 2011 August 18

\section{ABSTRACT}

We present the results of a gravitational lensing analysis of the bright $z_{\mathrm{s}}=2.957$ submillimeter galaxy (SMG) HERMES found in the Herschel/SPIRE science demonstration phase data from the Herschel Multi-tiered Extragalactic Survey (HerMES) project. The high-resolution imaging available in optical and near-IR channels, along with $\mathrm{CO}$ emission obtained with the Plateau de Bure Interferometer, allows us to precisely estimate the intrinsic source extension and hence estimate the total lensing magnification to be $\mu=10.9 \pm 0.7$. We measure the half-light radius $R_{\text {eff }}$ of the source in the rest-frame near-UV and $V$ bands that characterize the unobscured light coming from stars and find $R_{\mathrm{eff}, *}=[2.0 \pm 0.1] \mathrm{kpc}$, in good agreement with recent studies on the SMG population. This lens model is also used to estimate the size of the gas distribution $\left(R_{\text {eff,gas }}=[1.1 \pm 0.5] \mathrm{kpc}\right)$ by mapping back in the source plane the $\mathrm{CO}(J=5 \rightarrow 4)$ transition line emission. The lens modeling yields a relatively large Einstein radius $R_{\text {Ein }}=44^{\prime \prime} 10 \pm 0^{\prime} \cdot 02$, corresponding to a deflector velocity dispersion of [483 \pm 16$] \mathrm{km} \mathrm{s}^{-1}$. This shows that HERMES is lensed by a galaxy group-size dark matter halo at redshift $z_{1} \sim 0.6$. The projected dark matter contribution largely dominates the mass budget within the Einstein radius with $f_{\mathrm{dm}}\left(<R_{\mathrm{Ein}}\right) \sim 80 \%$. This fraction reduces to $f_{\mathrm{dm}}\left(<R_{\mathrm{eff}, \mathrm{G} 1} \simeq 4.5 \mathrm{kpc}\right) \sim 47 \%$ within the effective radius of the main deflecting galaxy of 
stellar mass $M_{*, \mathrm{G} 1}=[8.5 \pm 1.6] \times 10^{11} M_{\odot}$. At this smaller scale the dark matter fraction is consistent with results already found for massive lensing ellipticals at $z \sim 0.2$ from the Sloan Lens ACS Survey.

Key words: galaxies: elliptical and lenticular, $\mathrm{cD}$ - galaxies: groups: general - galaxies: halos - gravitational lensing: strong - submillimeter: galaxies

Online-only material: color figures

\section{INTRODUCTION}

The current generation of wide field surveys at submillimeter and millimeter wavelengths is now providing us with large numbers of high-redshift galaxies containing large amounts of dust heated by intense star formation or active galactic nucleus (AGN) activity (e.g., Hughes et al. 1998; Barger et al. 1998; Blain et al. 2002; Chapman et al. 2005; Coppin et al. 2006; Austermann et al. 2010). This population of submillimeter galaxies (SMGs) is easily detectable in the redshift range $1 \lesssim z \lesssim 5$ thanks to a strong negative $k$-correction when observed at wavelengths $\lambda \gtrsim 500 \mu \mathrm{m}$. This property, along with a sharp falloff at the bright luminosity end of their luminosity function, makes bright SMGs good candidates for being strongly gravitationally lensed (e.g., Blain 1996; Negrello et al. 2007, 2010; Vieira et al. 2010). Efficient identification of lensed SMGs is only now becoming possible thanks to surveys like the Herschel Multi-tiered Extragalactic Survey (HerMES) or the Herschel-Astrophysical Terahertz Large Area Survey (H-ATLAS) conducted with the Herschel satellite (e.g., Oliver et al. 2010; Griffin et al. 2010; Negrello et al. 2010) and from the ground with the South Pole Telescope (Vieira et al. 2010).

The interest of building large samples of lensed SMGs for getting better insights on the properties of these otherwise very faint objects is clear, and recent results are already shedding some light on the spatial distribution of gas, dust, and stars in these SMGs (e.g., Swinbank et al. 2010b). However, the redshift distribution of SMGs, which peaks in the range 2-2.5 (Chapman et al. 2005; Wardlow et al. 2011), is also well suited to probe the mass distribution of high-redshift deflectors, typically in the range $0.3<z_{1}<1.5$, which complements local studies like the Sloan Lens ACS Survey (SLACS) which are limited to $z \leqslant 0.4$ (Bolton et al. 2008; Auger et al. 2010).

In this paper, we present the modeling of the gravitationally lensed SMG HERMES, also referred to as HLSW-01, discovered in Herschel/SPIRE observations during the science demonstration phase by the HerMES program. Its J2000 coordinates are R.A. $=10: 57: 51.0$, decl. $=+57: 30: 25.8$, with a lens photometric redshift of $z_{1}=0.60 \pm 0.04$ (Oyaizu et al. 2008) and a source redshift of $z_{\mathrm{s}}=2.9575 \pm 0.0001$ (Riechers et al. 2011; Scott et al. 2011, hereafter R11 and S11). The goal of this lens modeling work is twofold. First, we want to recover the intrinsic light distribution of the source while optimally taking advantage of the magnifying power of the deflector. Detailed investigations on the lensed source are developed in associated papers (Conley et al. 2011, hereafter C11; R11; S11). And second we want to probe the mass content of the foreground object which, given the large image separation of the multiple images, might be very massive.

\footnotetext{
* Some of the data presented herein were obtained at the W. M. Keck Observatory which is operated as a scientific partnership among the California Institute of Technology, the University of California, and the National Aeronautics and Space Administration. The Observatory was made possible by the generous financial support of the W. M. Keck Foundation.

${ }^{35}$ Hubble Fellow.
}

The paper is thus organized as follows. In Section 2, we present the lens modeling techniques and the optical and nearIR (NIR) data we shall use along with the main results. The best-fit lens model is then used in Section 3 to reconstruct the source $\mathrm{CO}(J=5 \rightarrow 4)$ light distribution at $576 \mathrm{GHz}$ observed with the Plateau de Bure Interferometer (PdBI). In Section 4, we interpret the lens model results to measure the balance of dark and luminous matter in the inner $30 \mathrm{kpc}$ of the deflector. We conclude in Section 5. Throughout, we assume a concordance cosmology with matter and dark energy density $\Omega_{\mathrm{m}}=0.3$, $\Omega_{\Lambda}=0.7$, and Hubble constant $H_{0}=70 \mathrm{~km} \mathrm{~s}^{-1} \mathrm{Mpc}^{-1}$. Magnitudes are expressed in the AB system.

\section{LENS MODELING OF OPTICAL DATA}

\subsection{Observations}

For accurate lens modeling, we use the best spatial resolution images of sufficient signal to noise that are currently available for HLSW-01. A $1400 \mathrm{~s}$ image of the system was taken using Laser Guide Star Adaptive Optics in the $K_{\mathrm{p}}$ band with the NIRC2 instrument mounted on the Keck II Telescope. ${ }^{36}$ The observing conditions allowed us to achieve a typical 0'.1 FWHM pointspread function. The FWHM is well sampled with a plate scale of 0 ' 02 . We additionally use a one-hour Subaru SuprimeCam $i$-band observation with $\sim 0$ '.74 FWHM seeing and 0'.202 pixel size.

A $K_{\mathrm{p}}$ image of the central region of the system is shown in Figure 1 . The lensing configuration can easily be described despite the relatively low surface brightness of the multiply imaged features of HLSW-01. The configuration of multiple images lifts any ambiguities as we clearly see four images of similar surface brightness forming a so-called fold configuration with images 1 and 2 presumably merging through the critical line and images 3 and 4 being lower magnification conjugate images (see, e.g., Schneider et al. 1992 for a description of catastrophe theory in the context of gravitational lensing). A closer look at the $K_{\mathrm{p}}$-band image shows that image 1 is perturbed by a small galaxy, G4, which seems to be massive enough to split image 1 into two pieces (1a and $1 \mathrm{~b}$ ) on both sides of G4. Galaxies G2 and G3 may also act as potential perturbers on the innermost multiple image 4. To a lesser extent, G5 might also be considered as a perturbing galaxy. G1 is the central galaxy of the massive deflecting structure, which presumably should be a group of galaxies, given the large $\Delta \theta \simeq 8^{\prime \prime}$ image separation. Having no redshift information at all for these perturbers, the simplest assumption is to consider them to be at the same redshift as G1, keeping in mind that changes from this hypothesis are of second order in the lens modeling. Indeed, the total magnification of HLSW-01 is not changed, while the absolute mass calibration of $\mathrm{G} 2, \ldots, \mathrm{G} 5$ depends on the assumption that they are at the same redshift as G1. However, measuring the mass of perturbers is not the main motivation of the modeling.

\footnotetext{
$\overline{36}$ http://www2.keck.hawaii.edu/realpublic/inst/nirc2/index.html
} 


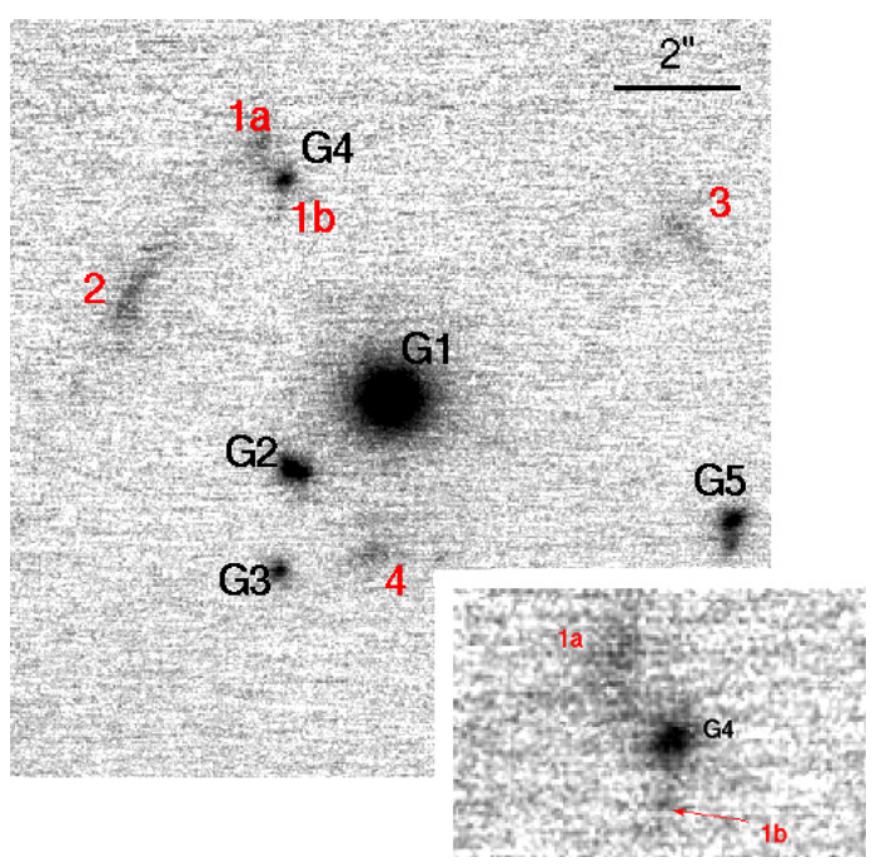

Figure 1. $K_{\mathrm{p}}$-band adaptive-optics-corrected overview of HLSW-01 with labeled multiple images (red) and foreground galaxies (black). North is up and east is left. The bottom right inset zooms into the image 1 that appears to be perturbed by the foreground galaxy G4 and split into two pieces.

(A color version of this figure is available in the online journal.)

\subsection{Method}

The lens modeling builds on the dedicated code sl_fit previously used for galaxy-scale strong lenses (e.g., Gavazzi et al. 2007, 2008; Ruff et al. 2011). It fits model parameters of simple analytic lensing potentials. sl_fit can be run in three different regimes of increasing computational cost. The first mode makes use of the coordinates of image plane points and minimizes the distance to their parent source plane locations in a way similar to gravlens (Keeton 2001) or lenstool (Kneib 1993; Jullo et al. 2007). The second mode uses the full surface brightness distribution and attempts to account for it with one or more simple analytic light components that we take to have a unique Gaussian radial profile with elliptical shape (see, e.g., Marshall et al. 2007 and Bolton et al. 2008 for similar techniques). Finally, the third mode implements a pixelized linear reconstruction of the source plane light distribution while fitting for the nonlinear potential parameters (Warren \& Dye 2003; Treu \& Koopmans 2004; Suyu et al. 2006); we did not consider the latter mode here as its computational cost is prohibitive for the large images and complex gravitational potential of this system.

The lensing potential is assumed to be made of a cored isothermal ellipsoid, centered on the main deflector galaxy G1, which is supposed to capture the lensing contribution of the dark matter halo as well as the stellar component of G1. Given the absence of a radial arc or central demagnified images (see, e.g., Gavazzi et al. 2003), the details of the assumed potential in the innermost parts $\left(r \lesssim 2^{\prime \prime}\right)$ of the lens should not be important. The peak of G1's light distribution is assumed to be the center of this potential component. The convergence profile of the central mass component is given by

$$
\kappa_{\text {cent }}(\vec{\xi})=\frac{b_{\text {cent }}}{2} \frac{1}{\sqrt{\xi^{2}+r_{c}^{2}}},
$$

where the scaling parameter $b_{\text {cent }}$ is related to the velocity dispersion of the deflector through $b / 1^{\prime \prime}=\left(\sigma_{v} / 186.21 \mathrm{~km} \mathrm{~s}^{-1}\right)^{2}$ $D_{\mathrm{ls}} / D_{\mathrm{s}}$, the core radius is $r_{c}, \xi^{2}=q x^{2}+y^{2} / q$ is the radial coordinate that accounts for the ellipsoidal symmetry of the isodensity contours, and $q$ is the minor-to-major axis ratio. The orientation of the major axis, $\theta_{\text {cent }}$, relative to the $x$ axis is allowed to vary, although this is not explicit in the definition of $\vec{\xi}$. As will be seen below, lens modeling of extended images can yield formal errors on $b$ of order $1 \%$, and therefore similar errors on $\sigma_{v}$. However, here we propagate uncertainties in $D_{\mathrm{ls}} / D_{\mathrm{s}}$ due to the relatively poorly known lens redshift $\left(z_{1}=0.60 \pm 0.04\right)$. This results in a dominant additional $3 \%$ error that we add in quadrature to model uncertainties on $b$, keeping in mind that a spectroscopic measurement of $z_{1}$ would readily reduce lens modeling errors on $\sigma_{v}$ to the percent level.

We carefully account for the perturbing galaxies G2, G3, G4, and the more distant G5, and model them as truncated isothermal ellipsoids, each with a free velocity dispersion and a constant cutoff radius that we fix to a value of $r_{\mathrm{t}}=1$ 1".5 corresponding to 10 kpc (see, e.g., Limousin et al. 2007, 2009; Suyu \& Halkola 2010). For each of these galaxies the convergence profile takes the form

$$
\kappa_{\text {pert }}\left(\vec{r}-r_{\mathrm{G}, \mathrm{i}}\right)=\frac{b_{\text {pert,i }}}{2}\left(\frac{1}{\xi}-\frac{1}{\sqrt{\xi^{2}+r_{t}^{2}}}\right),
$$

so that the total mass of a perturber is $M_{\text {pert,i }}=\pi \Sigma_{\text {crit }} b_{\text {pert }, \mathrm{i}} r_{t}$, where $\Sigma_{\text {crit }}$ is the critical surface density, which for this system has a value of $\Sigma_{\text {crit }} \simeq[1.80 \pm 0.05] \times 10^{9} M_{\odot} \mathrm{kpc}^{-2} \simeq$ $[8.05 \pm 0.24] \times 10^{10} M_{\odot} \operatorname{arcsec}^{-2}$. The $3 \%$ quoted errors are dominated by the uncertainty in the photometric redshift of the lens, but this is still negligible compared to the uncertainty in the velocity dispersion of the main component. As an approximation, we match the ellipticity and orientation of these perturbing systems to that of the stars they host, although we note that recent analyses have shown that departures from this simple assumption might occur and are sometimes observed with more favorable lensing configurations and deep space-based optical data (Suyu \& Halkola 2010).

To summarize, the lensing potential is described by four parameters for the central component: $b_{\text {cent }}, r_{c}, q_{\text {cent }}, \theta_{\text {cent }}$, and we also allow the four scaling parameters $b_{\text {pert, } i=2, \ldots, 5}$ of the perturbing potentials to vary.

\subsection{Preliminary Analysis: Conjugate Points}

We first attempted to fit the model parameters of the main central component assuming that the source is point-like (first mode of sl_fit) and the perturbers have a negligible effect. By ignoring the effect of G4 we had to neglect the additionally produced image $1 \mathrm{~b}$ and just considered the brightest pixel of each of the images 1a, 2, 3, and 4 as input constraints on the potential. We performed a fit to the parameters $q_{\text {cent }}, \theta_{\text {cent }}$, $b_{\text {cent }}$ and $r_{c}$. We used these results to further understand the source structure (fainter spots) seen in image 2 and how it is cast onto other images $1 \mathrm{a}, 3$, and 4 , respectively, and, hence increases the number of constraints (see, e.g., Gavazzi et al. 2003 for an illustration of the process). This also allowed us to determine which part of the source should be inside the extra caustic caused by the perturber G4 and thus seen as image 1b. Altogether we identified four multiply imaged knots. The error in positioning the knots is about 0 ' 1 , except for image $1 \mathrm{a}$, which we downweight with an error of 0.3 in order to minimize the 


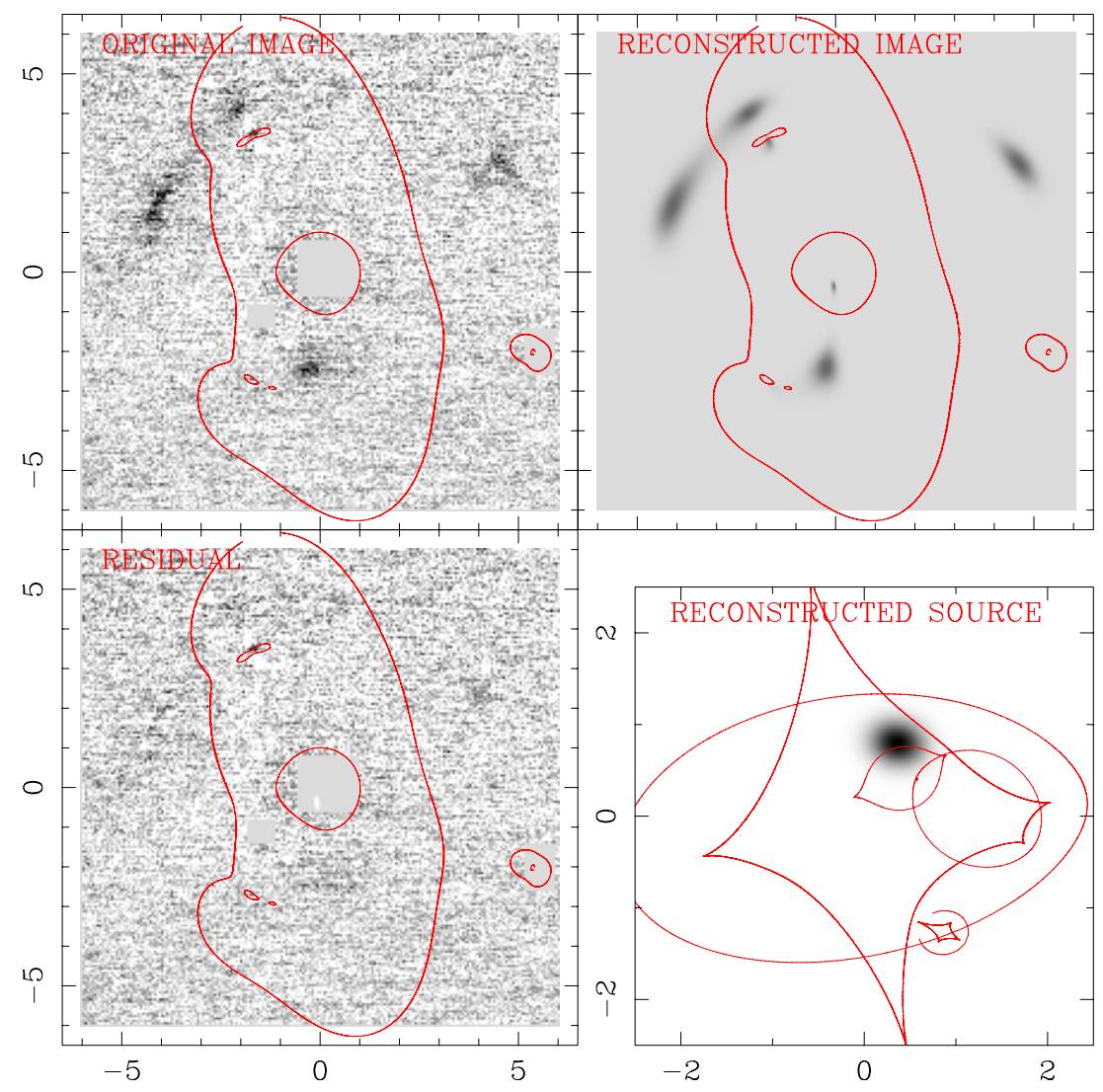

Figure 2. Results of the surface brightness fit of the Keck LGSAO $K_{\mathrm{p}}$-band imaging. Top left panel: input image with the foreground deflectors subtracted off (with their core completely masked out in some cases) using galf it. Top right panel: image plane model predictions. Bottom left panel: image plane residuals (data model). Bottom right panel: source plane model predictions. In this latter panel, the caustic lines are shown in red, whereas the other panels show the image plane critical lines. Scales are given in arcseconds.

(A color version of this figure is available in the online journal.)

sensitivity of the primary lens model parameters on the perturber G4 for this preliminary analysis.

We ran a Monte Carlo Markov Chain sampler of the posterior distribution of the eight model parameters related to the gravitational potential. At this stage the most important results ${ }^{37}$ are that we find quite an elongated mass distribution with axis ratio $q_{\text {cent }}=0.41 \pm 0.03$ and orientation $\theta_{\text {cent }}=13.8 \pm 0.5$ (north to east counterclockwise). The best-fit model has a $\chi^{2} /$ degree of freedom $($ dof $) \simeq 1.04$ per degree of freedom. The circularized Einstein radius is $R_{\text {Ein }}=44^{\prime \prime} 02 \pm 00^{\prime \prime} 05$, corresponding to a velocity dispersion of $\sigma_{v}=[473 \pm 15] \mathrm{km} \mathrm{s}^{-1}$, which suggests that the deflector is a massive group of galaxies. The modeling seems to require a finite core radius $r_{c}=$ $[2.0 \pm 1.0] \mathrm{kpc}$, but it is difficult to interpret this as a constraint on the dark matter distribution since the central component of Equation (1) captures both the contribution of stars and dark matter. The mass of perturber G4 is relatively well constrained: $M_{\mathrm{G} 4}=[25 \pm 5] \times 10^{10} M_{\odot}$, whereas $M_{\mathrm{G} 2}=[13 \pm 9] \times 10^{10} M_{\odot}$, $M_{\mathrm{G} 3}=[64 \pm 6] \times 10^{10} M_{\odot}$, and $M_{\mathrm{G} 5}=[20 \pm 12] \times$ $10^{10} M_{\odot}$. G5 has a nearly negligible effect on the lensing configuration.

This best-fit model predicts local magnifications ${ }^{38}$ of -1.87 , $4.37,2.57$, and -1.86 for images $1 \mathrm{~b}, 2,3$, and 4 , respectively, which means that the total magnification should be of order

\footnotetext{
37 For a given parameter we quote the median and $68 \%$ confidence level errors as given by the 16th and 84th percentiles of the marginalized distribution.

38 Negative values indicate a change of image parity with respect to that of the source.
}

10 for a point-like source. However, the above treatment of point-like lensing observables, although it allowed us to quickly explore the space of lens potential parameters, does not take full advantage of the spatially resolved surface brightness distribution. It thus cannot give us a clear idea of the intrinsic source light distribution on which the actual magnification factor depends.

\subsection{Keck LGSAO $K_{p}$-band Light Distribution}

We therefore placed an extended source with an analytic light profile made of an elliptical Gaussian with free central position $\left(x_{\mathrm{s}}, y_{\mathrm{s}}\right)$, axis ratio $\left(q_{\mathrm{s}}\right)$, position angle $\theta_{\mathrm{s}}$, flux $F_{\mathrm{s}}$, and half-light radius $R_{\text {eff,s. }}$. We optimized these parameters along with the potential parameters already introduced in the previous exploration phase using the surface-brightness mode of sl_fit. Figure 2 shows the resulting fit in the image plane. The corresponding reconstructed source is shown in the bottom right panel. The best fit is achieved with an extended source of $K_{\mathrm{p}}$-band magnitude $22.12 \pm 0.08$ and half-light radius $R_{\text {eff,s }}=[1.88 \pm 0.09] \mathrm{kpc}$.

We estimate the net magnification experienced by the source through a direct numerical sum of pixel values in the image and the source planes and find the magnification to be $\mu=$ $10.86 \pm 0.68$ after marginalizing over source and potential parameters. We show in Figure 3 the change of the magnification as a function of the source size $R_{\text {eff,s }}$. This suggests that, provided the size stays within a factor of $\sim 2$ for one band to another, the systematic change in magnification remains within 


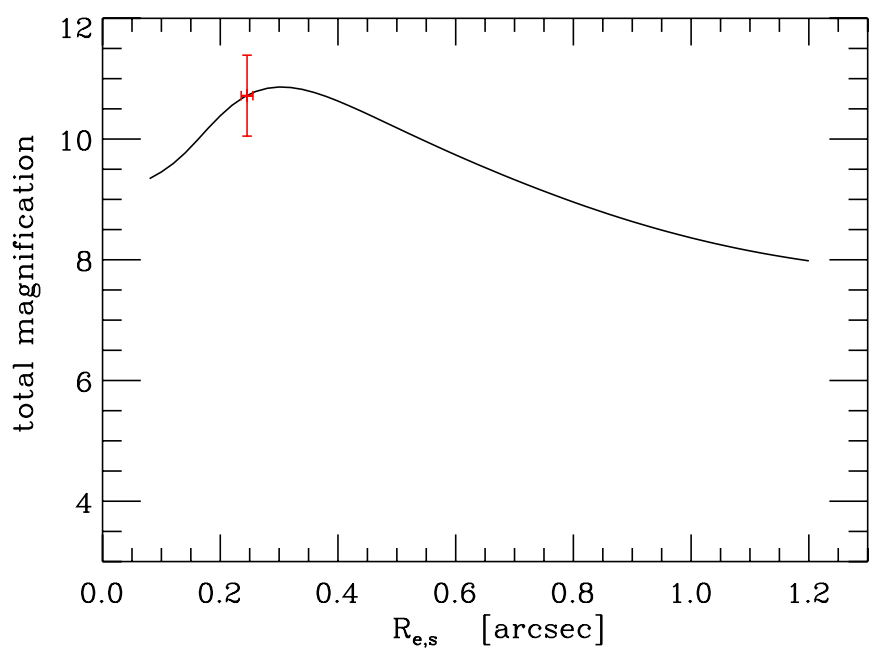

Figure 3. Change of the total magnification as a function of the characteristic source size $R_{\text {eff,s. }}$. The error bar shows the statistical error inferred from fitting the $K_{\mathrm{p}}$-band Keck LGSAO image (corresponding to a rest-frame $V$-band observation).

(A color version of this figure is available in the online journal.)

the current statistical errors. We can also see that magnification is shown to be $\lesssim 11.5$ whatever the source size and this can readily be cast as a robust lower bound on the intrinsic source flux for a given total observed flux.

Compared to the previous analysis using the conjugation of bright knots, the fit of an extended source yields little change in the recovered lens potential. The best-fit model yields results with a reduced $\chi^{2} /$ dof $\simeq 1.02$, although the number of free parameters is large $(201 \times 201$ pixels $)$ in the $K_{\mathrm{p}}$ image. The key features of the lens potential are as follows.

1. The axis ratio of the mass distribution is $q_{\text {cent }}=0.45 \pm 0.02$ with orientation $\theta_{\text {cent }}=11.9 \pm 0.4$.

2. The (circularized) Einstein radius is $R_{\text {Ein }}=4^{\prime \prime} .10 \pm 0$ '.02, which corresponds to a velocity dispersion of $\sigma_{v}=[483 \pm$ 16] $\mathrm{km} \mathrm{s}^{-1}$, in agreement with the preliminary study of Section 2.3. The mass content of this deflecting component is further discussed in Section 4.

3 . The core radius $r_{c}=[2.7 \pm 0.8] \mathrm{kpc}$ is found to be slightly larger. It is mostly constrained by the size and shape of the innermost image 4.

4. There is little change in the mass of perturbers, for which we find: $M_{\mathrm{G} 4}=[26.8 \pm 1.8] \times 10^{10} M_{\odot}, M_{\mathrm{G} 2}=[3.2 \pm$ $2.4] \times 10^{10} M_{\odot}, M_{\mathrm{G} 3}=[49.4 \pm 5.2] \times 10^{10} M_{\odot}$, and $M_{\mathrm{G} 5}=[12.7 \pm 7.0] \times 10^{10} M_{\odot}$.

\subsection{Seeing-limited Subaru i-band Light Distribution}

We now take advantage of the somewhat deeper seeinglimited Subaru $i$-band image to investigate the ability of the $K_{\mathrm{p}}$ model to account for observations at shorter wavelengths. This image corresponds to rest-frame $\sim 2000 \AA$ near-UV (NUV) emission that we expect to be lumpier and severely obscured by dust, and thus not to have the same extent as the rest-frame $V$-band probed by the $K_{\mathrm{p}}$-band image.

We therefore repeated the previous analysis on the Subaru $i$ image, but we considered the best-fit potential values above and only attempted to fit for the $i$-band light profile parameters of the source: central position $\left(x_{\mathrm{s}}, y_{\mathrm{s}}\right)$, axis ratio $\left(q_{\mathrm{s}}\right)$, position angle $\theta_{\mathrm{s}}$, flux $F_{\mathrm{s}}$, and half-light radius $R_{\mathrm{eff}, \mathrm{s}}$. Figure 4 shows the result

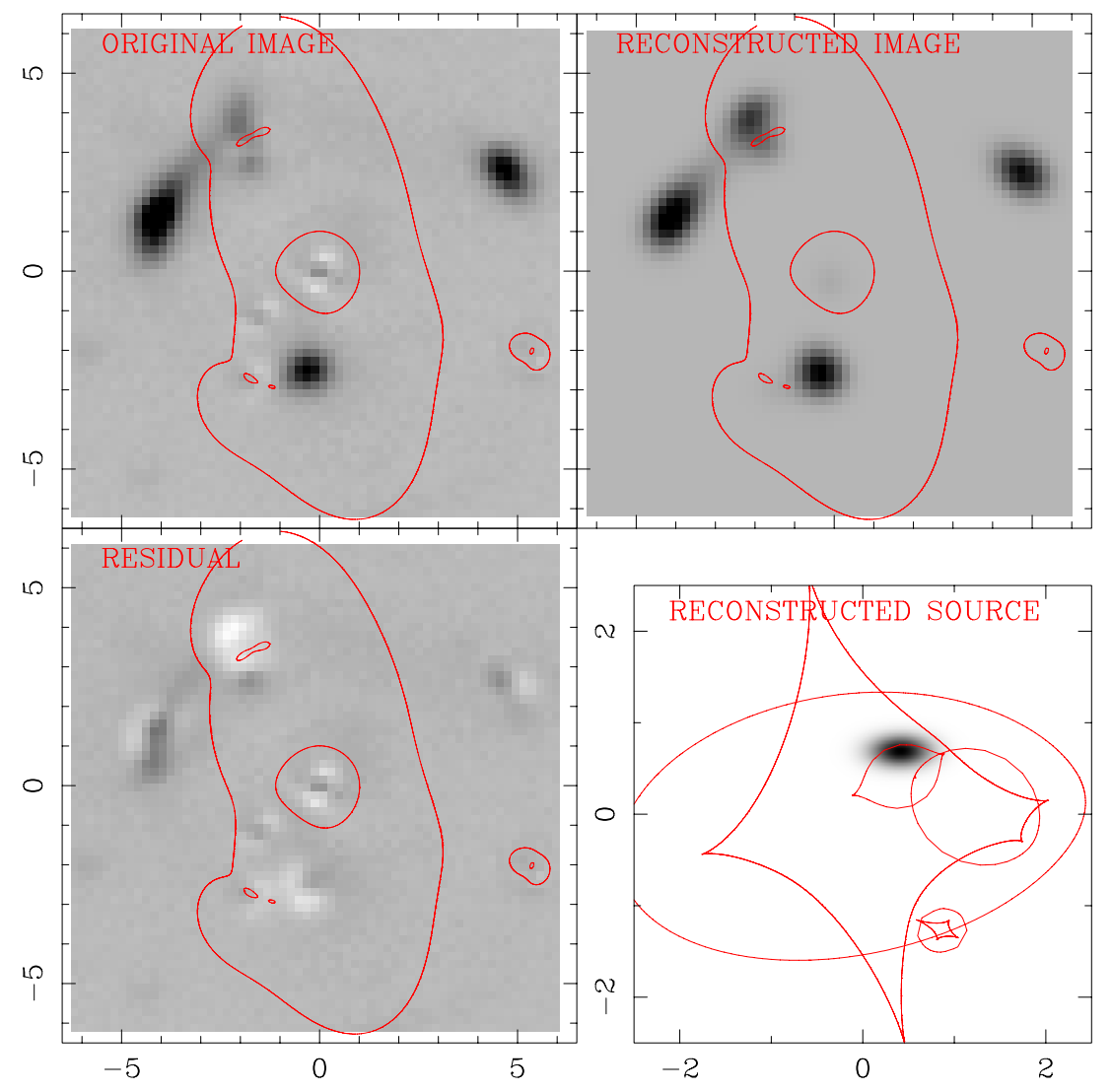

Figure 4. Subaru $i$-band modeling results. Top left panel: input image with the foreground deflectors subtracted off. Top right panel: image plane model predictions. Bottom left panel: image plane residuals (data model). Bottom right panel: source plane model predictions.

(A color version of this figure is available in the online journal.) 


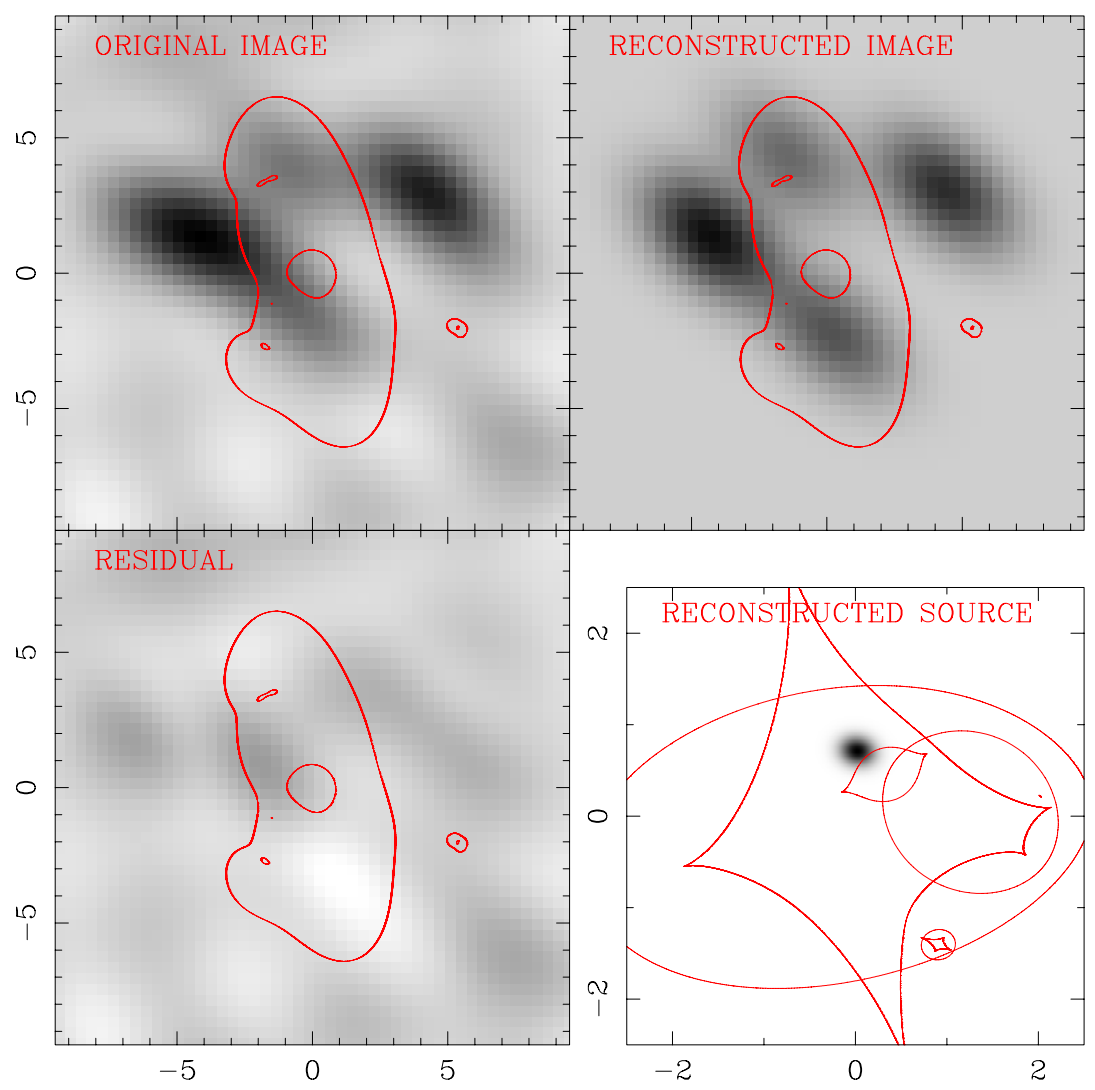

Figure 5. PdBI $\mathrm{CO}(J=5 \rightarrow 4)$ line flux density maps modeling results. Top left panel: input image with the foreground deflectors subtracted off. Top right panel: image plane model predictions. Bottom left panel: image plane residuals (data model). Bottom right panel: source plane model predictions. Note the slightly larger scale compared to Figures 2 and 4.

(A color version of this figure is available in the online journal.)

of the surface brightness distribution fit in the image plane. The corresponding reconstructed source is shown in the bottom right panel. The source is found to have a similar appearance in $i$ and $K_{\mathrm{p}}$ bands with a half-light radius $R_{\mathrm{eff}, \mathrm{s}}=[2.00 \pm 0.01] \mathrm{kpc}$ and an $i$-band AB magnitude of $22.72 \pm 0.01$. These small statistical errors should be treated with caution, as the residuals are worse than for the $K_{\mathrm{p}}$ case (the reduced $\chi^{2} /$ dof $\simeq 8$ per degree of freedom), which might indicate a more complex intrinsic NUV light distribution. These departures from a simple Gaussian elliptical profile could not be observed in $K_{\mathrm{p}}$ because of the substantially lower signal-to-noise ratio.

This latter analysis demonstrates that lens model parameters found by fitting the light distribution in the redder $K_{\mathrm{p}}$ filter give satisfying results for the fit of the light distribution in a different band, $i$.

\section{CO(5-4) LINE DISTRIBUTION}

Given the above success at accounting for the lensed visible/ NIR light distribution of HLSW-01, we extend our analysis to submillimeter wavelengths using spatially resolved PdBI observations of the $\mathrm{CO}(J=5 \rightarrow 4)$ transition line at $576 \mathrm{GHz}$ (see R11 for details).

Since our lens modeling code was originally designed to model optical/NIR images, we could not fit these observations directly in the $u v$-plane, which would allow a more careful handling of the beam convolution and limit the effect of noise correlations. In order to circumvent these difficulties we used a reduced image that was CLEANed with a synthesized beam of $4.8 \times 2$ ". 7 FWHM, with a major axis oriented +51.6 east of north. The measured noise rms is $1.2 \mathrm{mJy} \mathrm{beam}^{-1}$.

We did not attempt to fit for lens potential model parameters, as they were better determined with visible/NIR data, but we fit for the source position integrated intrinsic flux and shape. Figure 5 shows the result of the $\operatorname{CO}(J=5 \rightarrow 4)$ emission map fit in the image plane. The corresponding reconstructed source is shown in the bottom right panel. We can see that the modeling yields very small residuals beyond the secondary lobes that cannot be captured by our direct space modeling strategy. Similar to $K_{\mathrm{p}}$ imaging, we find a satisfactory best-fit result with a reduced $\chi^{2} /$ dof $\simeq 0.89$ (note however that the goodness of fit would accurately evaluated in the $u v$-plane). Our main inferences for the source parameters are as follows.

1. We find the source half flux radius to be $R_{\text {eff, }}=[1.13 \pm$ 0.53 ] kpc, slightly smaller than the extent we found for the rest-frame $V$ - and NUV-band fits.

2. We measure a small offset between the center of the gas distribution as probed by the $\mathrm{CO}(J=5 \rightarrow 4)$ transition and the stars that emit at visible/NIR wavelengths. The typical offset is $\Delta$ R.A. $=-0^{\prime \prime} \cdot 29 \pm 0.07$ and $\Delta$ decl. $=$ $-0{ }^{\prime} \cdot 10 \pm 0.05$ (corresponding to about $2.4 \mathrm{kpc}$ ). This suggests that rest-frame UV and visible light are severely obscured by the large dust content presumably associated with the gas revealed by the $\mathrm{CO}(J=5 \rightarrow 4)$ line (R11). The presence of dust is confirmed by the far-IR emission (C11). We stress however that further investigation of this is left for future work, including higher resolution millimeter or radio data. 
3. The intrinsic source luminosity of the $\operatorname{CO}(J=5 \rightarrow 4)$ line is $L_{\mathrm{CO}(5-4)}^{\prime}=[3.76 \pm 0.44] \times 10^{10} \mathrm{~K} \mathrm{~km} \mathrm{~s}^{-1} \mathrm{pc}^{2}$ including the equally important instrumental and modeling error terms.

The good signal-to-noise ratio of the PdBI data suggests that one could use the kinematical information to try and reconstruct the intrinsic velocity field as traced by the $\operatorname{CO}(J=$ $5 \rightarrow 4$ ) transition line. However, because of insufficient spatial resolution and perhaps nontrivial patterns in the velocity field, we were not able to explain the marginally resolved velocity field presented in Figure 5 of R11.

\section{MASS CONTENT OF THE DEFLECTOR}

We turn now to an analysis of our findings regarding the lens potential and compare the inferred mass distribution with the properties of the main deflector.

We used galfit (Peng et al. 2002) to measure the $K_{\mathrm{p}}$ light properties of the main galaxy G1 and found that the surface brightness profile is well fit by a Sérsic profile (Sersic 1968) of index $n=3.06 \pm 0.10$, effective radius $R_{\text {eff,G1 }}=$ $[4.5 \pm 0.1] \mathrm{kpc}$, total $K_{\mathrm{p}}$ magnitude $^{39} 17.41 \pm 0.10$, and a magnitude of $18.37 \pm 0.04$ in the $i$ band. Assuming a spectral energy distribution typical of an elliptical galaxy (Coleman et al. 1980), we can transform this observed magnitude into a restframe $V$-band luminosity $L_{V}=[3.9 \pm 0.6] \times 10^{11} L_{\odot}$, including errors on photometry $(4 \%)$, photometric redshift $(16 \%)$, and uncertainties in filter conversions (13\%). Taking into account the luminosity decline due to the passive evolution of its old stellar populations (Treu et al. 2001),

$$
\frac{d}{d z} \log \frac{M_{*}}{L_{V}} \simeq-0.40 \pm 0.05,
$$

we can predict the evolution-corrected luminosity. Applying the same stellar mass-to-light ratio as found by Gavazzi et al. (2007) for massive early-type galaxies in the SLACS survey, we get a total stellar mass of $M_{*, \mathrm{G} 1}=8.5 \pm 1.6 \times 10^{11} M_{\odot}$ for the central galaxy. This large stellar mass, along with the large characteristic velocity dispersion inferred from lens modeling $\sigma_{v}=[483 \pm 16] \mathrm{km} \mathrm{s}^{-1}$, suggests that the deflector is likely a massive group of galaxies with G1 being its dominating central galaxy. ${ }^{40}$

It is tempting to compare these results with the SLACS findings for massive early-type galaxies at redshift $z \sim 0.2$ and with median stellar mass $\sim 2-4 \times 10^{11} M_{\odot}$ (depending on the choice of the stellar initial mass function; Auger et al. 2010). We find that our lens model requires a finite core radius $r_{c}=[2.72 \pm 0.84] \mathrm{kpc}$. This is substantially different from lensing findings in $z \sim 0.2$ elliptical galaxies of slightly lower mass (e.g., Bolton et al. 2008; Koopmans et al. 2009) in which a singular isothermal elliptical profile provides a good description of the total lensing potential. In addition, the lensing-inferred velocity dispersion is unlikely to reflect the stellar velocity dispersion of G1 that would be measured by optical/NIR spectroscopy. Indeed, the fundamental plane (FP) of early-type galaxies (Djorgovski \& Davis 1987; Faber et al. 1987) predicts

\footnotetext{
39 Photometry is quite uncertain with adaptive optics imaging, and calibration was made difficult due to uncertainties in conversion between the NIRC2 $K_{\mathrm{p}}$ filter and the reference Two Micron All Sky Survey $K_{\mathrm{s}}$ photometry.

40 We could not find any concentration of galaxies within several arcminutes probed by the Subaru image, further indicating that G1 is the main mass component.
}

that the velocity dispersion of G1 should be [400 \pm 30$] \mathrm{km} \mathrm{s}^{-1}$ with the latest normalization of Auger et al. (2010). However, the finite core radius requires a numerical integration of the Jeans equation to relate our total density profile inferred from lensing and a true velocity dispersion $\sigma_{\text {ap }}$ within a fiducial aperture of radius $R_{\mathrm{ap}}=11^{\prime \prime} .5 \simeq 10 \mathrm{kpc}$. Following the prescriptions of Mamon \& Łokas (2005) for fast numerical integrations of the Jeans equation, assuming an isotropic velocity tensor, our model predicts a value of $\sigma_{\mathrm{ap}}=[380 \pm 5] \mathrm{km} \mathrm{s}^{-1}$, in good agreement with the FP value.

A direct comparison with SLACS is complicated by the very different values of Einstein radii. For SLACS the ratio is $R_{\text {Ein }} / R_{\text {eff }} \sim 0.5$ whereas we observe $R_{\text {Ein }} \sim 27 \mathrm{kpc} \sim 6 \times$ $R_{\text {eff }}$. Therefore, it is not surprising that the total mass within $R_{\text {Ein }}$ is a factor of $\sim 5$ greater than the stellar mass. ${ }^{41}$ The deflector is thus a group of galaxies that is more dark matter dominated than most SLACS massive early-type galaxies. We note that the cosmological critical density for lensing $\Sigma_{\text {crit }}=$ $c^{2} /(4 \pi G) D_{\mathrm{s}} /\left(D_{\mathrm{ls}} D_{\mathrm{os}}\right)$ is more favorable for SMGs (with typical redshifts $z_{\mathrm{s}} \sim 2-3$ and deflectors at $z_{1} \sim 0.6$ ) than for SLACS lenses (for which $z_{\mathrm{s}} \sim 0.7$ and $z_{1} \sim 0.2$ ). Consequently, the deflector in HLSW-01 and similar $z \sim 0.6$ systems will typically have twice as large an Einstein radius as lower redshift SLACS lenses, regardless of differences in the deflector mass. This large radius implies that these lenses will presumably be more dark matter dominated. Extrapolating our best-fit density profile inward to the effective radius yields a projected dark matter fraction $f_{\mathrm{dm}}\left(<R_{\mathrm{eff}}\right)=0.47_{-0.26}^{+0.19}$, in agreement with SLACS findings.

\section{CONCLUSIONS}

We have calculated a detailed lens model of the newly found gravitational lens HERMES. Taking advantage of the best available spatial resolution images in the $K_{\mathrm{p}}$ band using the Laser Guide Star Adaptive Optics (LGSAO) system at the Keck II Telescope, in the $i$ band with the Subaru Telescope and using PdBI to probe the $\mathrm{CO}(J=5 \rightarrow 4)$ transition line at $576 \mathrm{GHz}$, we were able to infer the mass distribution in the inner $\sim 30 \mathrm{kpc}$ of the deflecting structure, which turns out to be a massive galaxy, presumably at the center of a galaxy group size halo of characteristic velocity dispersion $\sigma_{v}=[483 \pm 16] \mathrm{km} \mathrm{s}^{-1}$.

The redshift distribution of lensed SMGs will naturally select objects in a favorable range for producing large Einstein radii, compared to local lenses. This provides a great opportunity to probe the total density profile and the dark matter content of intermediate redshift halos with high accuracy. The lensing configuration of HLSW-01 yields a value of $R_{\text {Ein }}=4 . \prime 25 \sim 27 \mathrm{kpc}$ that corresponds to six times the effective radius of the central galaxy. At these scales we observe a projected dark matter fraction of about $80 \%$. The deflector of HLSW-01 probably stands in an interesting transition regime between cluster and galaxy scale lenses, in which a joint lensing plus dynamical analysis (Miralda-Escudé 1995; Sand et al. 2004; Gavazzi 2005; Newman et al. 2009) would be very informative for the actual small-scale dark matter distribution.

Much of the novelty of the large number of lensed SMGs to be uncovered in ongoing and future submillimetric surveys resides in the opportunity for studying faint heavily star-forming galaxies with good resolution and signal to noise that would otherwise be unreachable. In the particular case of HLSW-01,

\footnotetext{
41 This corresponds to a total mass-to-light ratio of

$M_{\mathrm{tot}} / L_{V}=18.0_{-2.7}^{+3.6}\left(M / L_{V}\right)_{\odot}$, again, correcting for luminosity evolution.
} 
the source turns out to be magnified by a factor $\sim 10$, which allowed us to measure the extent of the emitting gas in the $\mathrm{CO}(J=5 \rightarrow 4)$ transition line as well as young stars emitting in the NUV and visible bands. We measured a half-light radius $R_{\text {eff,gas }}=[1.13 \pm 0.53] \mathrm{kpc}$ and $R_{\text {eff, } *}=[2.0 \pm 0.1] \mathrm{kpc}$ using PdBI and Keck $K_{\mathrm{p}}$ data, respectively, in excellent agreement with the sample of SMGs studied by Swinbank et al. (2010a). There is some evidence that the peak of the $\mathrm{CO}$ emission and the peak of the NUV/visible light could be offset by $\sim 0.3 \simeq 2.4 \mathrm{kpc}$, which could be explained if the stellar light is obscured by dust associated with the cold gas. The source reconstruction allows for a careful estimate of the intrinsic source flux at many other wavelengths, thus allowing a more detailed analysis of the gas and dust content of this peculiar SMG (C11; R11; S11) and its gas content.

The large number of lensed SMGs like HLSW-01 to be found in the coming years with HerMES, H-ATLAS, the SPT, and other surveys, will allow detailed analyses of the central regions of massive galaxies with exquisite accuracy over a broad range of deflector redshifts. This is a good opportunity to constrain evolutionary trends in their dynamical properties, including clues on the role of dry versus wet mergers or the role of the central AGN. At the same time, the resolving power of gravitational lensing will allow detailed investigations of the stellar, gaseous, and dust content of massively star-forming SMGs at high redshift.

R.G. and A.O. acknowledge support from the Centre National de Etudes Spatiales. S.G.D. and A.A.M. acknowledge partial support from SN grant AST-0909182 and the Ajax Foundation. SPIRE has been developed by a consortium of institutes led by Cardiff University (UK) and including University of Lethbridge (Canada); NAOC (China); CEA, LAM (France); IFSI, University of Padua (Italy); IAC (Spain); Stockholm Observatory (Sweden); Imperial College London, RAL, UCL-MSSL, UKATC, University of Sussex (UK); Caltech, JPL, NHSC, University of Colorado (USA). This development has been supported by national funding agencies: CSA (Canada); NAOC (China); CEA, CNES, CNRS (France); ASI (Italy); MCINN (Spain); SNSB (Sweden); STFC (UK); and NASA (USA). The IRAM Plateau de Bure Interferometer is supported by INSU/CNRS (France), MPG (Germany), and IGN (Spain).

\section{REFERENCES}

Auger, M. W., Treu, T., Bolton, A. S., Gavazzi, R., Koopmans, L. V. E., Marshall, P. J., Moustakas, L. A., \& Burles, S. 2010, ApJ, 724, 511

Austermann, J. E., et al. 2010, MNRAS, 401, 160

Barger, A. J., Cowie, L. L., Sanders, D. B., Fulton, E., Taniguchi, Y., Sato, Y., Kawara, K., \& Okuda, H. 1998, Nature, 394, 248

Blain, A. W. 1996, MNRAS, 283, 1340
Blain, A. W., Smail, I., Ivison, R. J., Kneib, J., \& Frayer, D. T. 2002, Phys. Rep., 369,111

Bolton, A. S., Burles, S., Koopmans, L. V. E., Treu, T., Gavazzi, R., Moustakas, L. A., Wayth, R., \& Schlegel, D. J. 2008, ApJ, 682, 964

Chapman, S. C., Blain, A. W., Smail, I., \& Ivison, R. J. 2005, ApJ, 622, 772

Coleman, G. D., Wu, C.-C., \& Weedman, D. W. 1980, ApJS, 43, 393

Conley, A., et al. 2011, ApJ, 732, L35

Coppin, K., et al. 2006, MNRAS, 372, 1621

Djorgovski, S., \& Davis, M. 1987, ApJ, 313, 59

Faber, S. M., Dressler, A., Davies, R. L., Burstein, D., \& Lynden-Bell, D. 1987, in Proc. 8th Santa Cruz Summer Workshop in Astronomy and Astrophysics, Nearly Normal Galaxies: From the Planck Time to the Present, ed. S. M. Faber (New York: Springer), 175

Gavazzi, R. 2005, A\&A, 443, 793

Gavazzi, R., Fort, B., Mellier, Y., Pelló, R., \& Dantel-Fort, M. 2003, A\&A, 403, 11

Gavazzi, R., Treu, T., Koopmans, L. V. E., Bolton, A. S., Moustakas, L. A., Burles, S., \& Marshall, P. J. 2008, ApJ, 677, 1046

Gavazzi, R., Treu, T., Rhodes, J. D., Koopmans, L. V. E., Bolton, A. S., Burles, S., Massey, R. J., \& Moustakas, L. A. 2007, ApJ, 667, 176

Griffin, M. J., et al. 2010, A\&A, 518, L3

Hughes, D. H., et al. 1998, Nature, 394, 241

Jullo, E., Kneib, J.-P., Limousin, M., Elíasdóttir, Á., Marshall, P. J., \& Verdugo, T. 2007, New J. Phys., 9, 447

Keeton, C. 2001, arXiv:astro-ph/0102340

Kneib, J.-P. 1993, PhD thesis, Univ. Toulouse

Koopmans, L. V. E., et al. 2009, ApJ, 703, L51

Limousin, M., Kneib, J. P., Bardeau, S., Natarajan, P., Czoske, O., Smail, I., Ebeling, H., \& Smith, G. P. 2007, A\&A, 461, 881

Limousin, M., Sommer-Larsen, J., Natarajan, P., \& Milvang-Jensen, B. 2009, ApJ, 696, 1771

Mamon, G. A., \& Łokas, E. L. 2005, MNRAS, 363, 705

Marshall, P. J., et al. 2007, ApJ, 671, 1196

Miralda-Escudé, J. 1995, ApJ, 438, 514

Negrello, M., Perrotta, F., González-Nuevo, J., Silva, L., de Zotti, G., Granato, G. L., Baccigalupi, C., \& Danese, L. 2007, MNRAS, 377, 1557

Negrello, M., et al. 2010, Science, 330, 800

Newman, A. B., Treu, T., Ellis, R. S., Sand, D. J., Richard, J., Marshall, P. J., Capak, P., \& Miyazaki, S. 2009, ApJ, 706, 1078

Oliver, S. J., et al. 2010, A\&A, 518, L21

Oyaizu, H., Lima, M., Cunha, C. E., Lin, H., Frieman, J., \& Sheldon, E. S. 2008, ApJ, 674, 768

Peng, C. Y., Ho, L. C., Impey, C. D., \& Rix, H. 2002, AJ, 124, 266

Riechers, D. A., et al. 2011, ApJ, 733, L12 (R11)

Ruff, A. J., Gavazzi, R., Marshall, P. J., Treu, T., Auger, M. W., \& Brault, F. 2011, ApJ, 727, 96

Sand, D. J., Treu, T., Smith, G. P., \& Ellis, R. S. 2004, ApJ, 604, 88

Schneider, P., Ehlers, J., \& Falco, E. E. 1992, Gravitational Lenses (Berlin: Springer)

Scott, K. S., et al. 2011, ApJ, 733, 29 (S11)

Sersic, J. L. 1968, Atlas de Galaxias Australes (Cordoba: Observatorio Astronomico)

Suyu, S. H., \& Halkola, A. 2010, A\&A, 524, A94

Suyu, S. H., Marshall, P. J., Hobson, M. P., \& Blandford, R. D. 2006, MNRAS, 371,983

Swinbank, A. M., et al. 2010a, MNRAS, 405, 234

Swinbank, A. M., et al. 2010b, Nature, 464, 733

Treu, T., \& Koopmans, L. V. E. 2004, ApJ, 611, 739

Treu, T., Stiavelli, M., Bertin, G., Casertano, S., \& Møller, P. 2001, MNRAS, 326,237

Vieira, J. D., et al. 2010, ApJ, 719, 763

Wardlow, J. L., et al. 2011, MNRAS, 415, 1479

Warren, S. J., \& Dye, S. 2003, ApJ, 590, 673 